\title{
Os custos do Programa de Saúde da Família de Porto Alegre - 1998 e 2002
}

\author{
The cost of the Family Health Program \\ in Porto Alegre - 1998 and 2002
}

\begin{abstract}
Resumo
Este estudo pretende, por meio da perspectiva das ciências econômicas, ampliar a compreensão sobre funcionamento do sistema de saúde, utilizando o instrumental disponível nesta área do conhecimento, as avaliações econômicas, para avaliar uma importante política pública proposta pelo governo federal e implantada em todo o país: o Programa de Saúde da Família. Nesta primeira aproximação será feito um estudo de caso do município de Porto Alegre, no período de 1998 a 2002. O objetivo do estudo é criar um instrumento de gestão dos custos do programa, que permita o monitoramento e redirecionamento das ações quando se fizer necessário. Foi feita extensa revisão da literatura e, para a construção do instrumento de gestão de custos, foi necessário o levantamento dos processos de trabalho, da estrutura gerencial da secretaria e por fim dos dados de despesa e produção para o calculo dos custos do programa. O estudo demonstrou que não houve variação importante dos custos totais do programa no período, mas houve grande variação em algumas categorias especificas de custo. Além disso, mostrou que as diferentes unidades de saúde possuem diferentes custos que estão relacionados com o tamanho da equipe ou com a população adscrita.
\end{abstract}

\begin{abstract}
This study aims at a broader understanding of the functioning of the health system from the perspective of economics, utilizing the tools made available by this area for evaluating an important public policy proposed by the Brazilian federal government: the Family Health Program. This first approach consists in a case study conducted in the city of Porto Alegre during the period 1998-2002. The purpose of this study was creating an instrument for the cost management of the program allowing for monitoring and redirecting actions when necessary. An extensive review of the literature was carried out and data about the work processes, the organizational structure of the secretariat in charge and cost and production of the program were collected for constructing the cost management instrument. The study showed no important variation in the total cost of the program during the period but considerable variations in some specific cost categories. Furthermore it was shown that different health units involve different costs related to the size of the team or to the target population.
\end{abstract}

Palavras-chave: Custos de Cuidados de Saúde; Economia de Saúde; Atenção Primária à Saúde.
Key Words: Health Care Cost; Health Economics;

Primary Health Care.

*Doutora em Saúde Coletiva, UNICAMP, MSc Health Planning and Financing, London School of Economics, Pesquisadora Visitante, Fundação Oswaldo Cruz, Rio de Janeiro, Brasil.

**Mestre em Administração, UFRGS, Analista de Sistemas, Datasus, Ministério da Saúde, Brasília, Brasil. 


\section{Introdução}

Esta pesquisa foi realizada no Programa de Saúde da Família (PSF) de Porto Alegre, retomando um estudo realizado em $1998^{1}$ que utilizou a metodologia para apuração de custos por absorção adaptada para calcular os custos da atenção básica no sistema público. Esta metodologia será aplicada neste estudo com o propósito de analisar as mudanças ocorridas nos custos, comparando os dados de 1998 e 2002.

A escolha por uma pesquisa nesta área foi o resultado de um contínuo e intenso envolvimento com discussões acerca da forma como as ciências econômicas, seus instrumentos e suas metodologias de trabalho específico poderiam ser utilizados para modificar a realidade, melhorando a qualidade e a eficiência dos serviços públicos de saúde no Brasil $2,3,4,5,6,7,8$.

Nos últimos anos houve uma grande preocupação por parte dos pesquisadores nacionais com a questão da avaliação, mas a questão da análise de custos e seus efeitos não têm sido abordados com a mesma intensidade, sendo esta uma lacuna a ser preenchida. Desta forma, o desenvolvimento desta área do conhecimento é muito importante, pois é preciso enfrentar a discussão da eficiência do uso dos recursos públicos, uma vez que estes são escassos e devem servir da melhor forma à sociedade. É preciso, portanto, enfrentar a discussão da eficiência do gasto como uma questão ética. Estudos que relacionam os "recursos empregados com os efeitos obtidos" são uma "modalidade de pesquisa avaliativa", pois procuram “estabelecer um julgamento ex-post de uma intervenção" ${ }^{\prime, 10}$. Os instrumentos econômicos de gerenciamento dos custos permitem conhecer melhor alguns aspectos da realidade dos serviços e conseqüentemente viabilizam a construção de soluções para cada situação-problema encontrada ${ }^{11}$ 12, 13, 14, 15, 16, 17 .

O conceito de avaliação econômica em saúde pode ser resumido como a análise comparativa entre diferentes alternativas em relação aos seus custos e resultados. Para que uma avaliação econômica seja considerada completa é necessário que contemple essa condição: realizar comparações entre duas ou mais alternativas possíveis em relação aos seus custos e resultados. A realização de uma avaliação econômica incompleta, no entanto, também é importante, pois é capaz de iniciar o processo e, mesmo sendo incompleta, identificar a melhor solução do ponto de vista econômico, subsidiando a decisão do gestor que levará em conta também outras variáveis $^{11,12,13,14,15,16,17}$.

O conhecimento da estrutura de custos de uma instituição é muito importante para o melhor gerenciamento dos serviços, pois racionaliza o uso dos insumos e utiliza estas informações para apoiar o planejamento a médio e longo prazo. Quando da aplicação de um sistema de custeio, a principal preocupação não deve ser apenas em relação à determinação dos preços, mas, principalmente, quanto a sua utilização enquanto fonte de informações para o controle, a avaliação e o planejamento das atividades das instituições ${ }^{18,19,20}$.

Estes sistemas podem considerar a totalidade dos gastos para a produção de determinado produto - neste caso, são chamados de sistemas de custeio integral - ou apenas parcela das despesas - neste caso são chamados de sistemas de custeio parcial. O grau de complexidade exigido pelos sistemas de custeio integral é maior; pois, nestes casos, é necessário considerar os custos indiretos e criar uma maneira de dividir estes custos entre os produtos ou serviços produzidos pela instituição. O sistema de custeio por absorção é considerado um sistema de custeio integral, pois ele apropria todos os custos incorridos para a produção de um bem ou serviço, ou seja, considera os custos diretos, indiretos, fixos e variáveis. Para a realização do cálculo de custo, utilizando-se o sistema de custeio por absorção, a primeira condição é a "setorização" ou divisão da instituição em centros de custos. As características principais dos centros de custos ou resultado são: serem uma unidade autônoma; possuírem uma área circunscrita de abrangência; serem fonte geradora de custos; possuírem identidade de objetivos comuns; terem uma unidade de mensuração específica; possuírem homogeneidade funcional e terem unidade de responsabililidade ${ }^{18,19,20}$.

O sistema de apropriação de custos por absorção é muito utilizado e pode trazer grandes vantagens para as instituições, principalmente para aquelas que não possuem 
ainda condições de informatizar todas as suas informações e cujo custo indireto não é preponderante no custo total. Como principal desvantagem do sistema de custeio por absorção é apontada pelos autores a dificuldade de se realizarem os rateios dos custos indiretos, que são feitos utilizando-se critérios arbitrários, o que implica, muitas vezes, avaliação incorreta dos custos finais ${ }^{18,19,20}$.

No Brasil, a proposta do Sistema Único de Saúde (SUS) é de que o planejamento seja usado para organizar e distribuir os serviços de saúde conforme a necessidade da população. Estes serviços são gratuitos, o atendimento é universal e organizado regionalmente. Os serviços são hierarquizados, conforme a sua complexidade tecnológica. A rede básica é a porta de entrada do sistema e deve garantir o atendimento ambulatorial básico e o desenvolvimento das ações de saúde pública. Neste modelo, a saúde é considerada um direito do cidadão.

O processo de descentralização da saúde, previsto quando da criação do SUS, tomou maior impulso a partir da promulgação da Constituição Federal em 1988 e da conseqüente aprovação das leis 8.080, de 19 de setembro de 1990, e a lei 8.142, de 28 de dezembro de 1990, as quais prevêem, entre outras coisas, a descentralização e o repasse de recursos financeiros para estados e municípios. Compondo esse conjunto de leis, está, também, a Norma Operacional Básica do SUS 01/93 (NOB/SUS 01/93), aprovada pela portaria MS n 545, de 20 de maio de 1993, que estabeleceu "normas e procedimentos reguladores do processo de descentralização da gestão das ações e serviços de saúde”. Foi a implantação da NOB/SUS 01/93 que permitiu a efetivação do processo de descentralização da saúde para o nível local no país.

Sendo assim, nas ultimas décadas, as mudanças no SUS, especialmente a descentralização de atribuições para os municípios sem a correspondente transferência de recursos financeiros ${ }^{1,18,19,20}$ e também a contínua absorção de novas e caras tecnologias pelo setor, trouxeram a discussão sobre custos da atenção para a ordem do dia. É cada vez mais importante para o gestor conhecer e controlar a sua estrutura de custos, especialmente se um de seus objetivos for garantir o atendimento de saúde para a população levando em conta os princípios do SUS da universalidade, integralidade e equidade ${ }^{21,22,23,24,25,26,27}$.

O Programa de Saúde da Família (PSF) ${ }^{28,29}$ é um programa prioritário do governo federal e pretende ser uma forma de organizar o sistema para garantir os preceitos constitucionais; sendo assim, é muito importante aumentar o nosso conhecimento sobre os custos deste programa; pois, como afirmam alguns autores ${ }^{23}$, a maior parte das instituições de saúde não conhece a sua estrutura de custos, pois não existe um "sistema técnico, detalhado e consistente de custos".

Em Porto Alegre os serviços de saúde do município estão divididos em duas áreas principais: a vigilância da saúde e a assistência à saúde. A vigilância da saúde é realizada pelo Centro de Vigilância em Saúde (CVS), e a atenção à saúde, por sua vez, tem a seguinte estrutura:

(a) Serviços de Atenção Ambulatorial Básica - são as unidades de saúde e as unidades do Programa de Saúde da Família. (b) Serviços de Atenção Ambulatorial Especializada compostos pelos serviços públicos: centros de saúde, ambulatórios especializados, unidades Psicossocial e os serviços privados contratados e conveniados.

(c) Atenção Hospitalar Contratada.

(d) Serviços de Emergência Próprios: o hospital de prontosocorro, os serviços de pronto-atendimento 24 horas e o SAMU, Serviço de Pronto Atendimento Pré-hospitalar.

A cidade foi dividida em distritos de saúde, e, em cada um, existe uma gerência local responsável pela organização dos serviços na região. As Unidades de Saúde que compõem a rede básica de saúde devem ser a porta de entrada do sistema. Nestas Unidades de Saúde, são oferecidos os serviços de uma equipe multidisciplinar composta por: (a) médicos (clínico geral, ginecologista e pediatra); (b) enfermeiros; (c) auxiliares de enfermagem; (d) nutricionistas. Também na rede básica estão as unidades de saúde do PSF. Nestas, a equipe é composta por: (a) médico generalista; (b) enfermeiros; (c) auxiliares de enfermagem; (d) agentes comunitários. Nas unidades de saúde do PSF, é definida a área de abrangência e as famílias da região são cadastradas. 
Os serviços realizados na rede básica são: (a) agendamento de consulta com especialista; (b) medicamento e curativos; (c) vacinas; (d) nebulizações; (e) teste do pezinho; (f) programa do leite; (g) programa de acompanhamento a crianças menores de um ano; (h) exame pré-natal e preventivo do câncer; (i) encaminhamentos para exames; (j) distribuição de camisinhas e anticoncepcionais; (l) injeções; (m) atividades em grupo com hipertensos, diabéticos, adolescentes e portadores do vírus HIV; (n) consultas - que podem ter sido agendadas ou podem ser atendimentos à demanda espontânea e às intercorrências. Nas unidades do PSF também são realizados atendimentos domiciliares e outras atividades em grupo na comunidade.

Quando houver necessidade de consultar um especialista, a unidade será responsável pelo agendamento da consulta por meio da central de marcação de consultas. Cada unidade dispõe de cotas mensais de exames, e os pacientes são encaminhados para os serviços contratados conforme necessidade e disponibilidade.

No município, a rede hospitalar mantém o vínculo com os níveis estadual e federal ou é terceirizada. O município gerencia a compra de serviços de internação hospitalar, inclusive parte das internações da região metropolitana e do interior do estado.

Em casos de emergência, devem ser procurados os serviços de pronto atendimento ou o serviço de atendimento pré-hospitalar, SAMU, que pode ser acionado pelo telefone 192, em caso de acidentes na rua.

Porto Alegre atende a demanda por serviços de saúde de sua população e também parte da demanda dos municípios da região metropolitana e do interior. Nestas circunstâncias, é difícil organizar, planejar e estabelecer o fluxo de pacientes no sistema.

Além dos serviços de atenção à saúde, a secretaria assumiu a Vigilância à Saúde no município e, atualmente, realiza o controle: (a) das águas; (b) das zoonoses; (c) dos produtos de interesse à saúde; (d) dos serviços de interesse à saúde; (e) epidemiológico; (f) da geração de informações em saúde.

O Programa de Saúde da Família foi criado pelo
Ministério da Saúde com a meta de capacitar 2.500 equipes, beneficiando 2,5 milhões de famílias pela implantação do PSF nas 26 unidades da Federação e $\mathrm{DF}^{28,29}$. As bases do Programa de Saúde da Família são: (a) construir um "modelo voltado para a proteção e promoção da saúde"; (b) trabalhar com "área de abrangência e adscrição de clientela" (entre 600 a 1000 famílias); (c) trabalhar com a equipe mínima composta por um médico, um enfermeiro, um auxiliar de enfermagem e de quatro a seis agentes comunitários; (d) a equipe deve residir na área de atuação; (e) deve haver a participação da comunidade; (f) o âmbito da atenção é integral e contínuo; $(\mathrm{g})$ deve ser a porta de entrada ao sistema único; (h) deve permitir o redirecionamento da formação dos profissionais de saúde; (j) deve haver remuneração diferenciada aos profissionais do PSF, e o controle social deve ser exercido pelo Conselho de Saúde e pelas comunidades. O objetivo geral do PSF é construir um novo modelo assistencial dirigido aos indivíduos, à família e à comunidade, além de ser fundamentado na promoção, proteção, diagnóstico precoce, tratamento e recuperação da saúde, de acordo com os princípios do SUS. As atividades da equipe do PSF podem ser resumidas em três grandes grupos: Atendimento na Comunidade, Atendimento nas Unidades de Saúde e a Referência e Contra-referência de pacientes ${ }^{28,29}$.

No grupo Atendimento nas Comunidades incluemse: (a) o diagnóstico da comunidade; (b) as visitas domiciliares; (c) a internação domiciliar; (d) a participação em grupos comunitários. No grupo Atendimento nas Unidades de Saúde estão os programas e a demanda espontânea. No grupo Referência e Contra-referência temos: (a) o apoio diagnóstico; (b) o atendimento em serviços ambulatoriais especializados; (c) a internação hospitalar; (d) a remoção de pacientes.

Estava previsto que o processo de recrutamento de recursos humanos, em um primeiro momento, deveria ser realizado com a assessoria dos níveis federal e estadual; assim como a realização de supervisão e avaliação do programa, e a implantação do Sistema de Informação (SIAB) específico com informações sobre: o cadastro familiar, o cartão de identificação, o prontuário familiar e o registro dos atendimentos. Assim, seria formado um banco de 
dados que permitiria a construção de indicadores em nível nacional. O financiamento do PSF deveria ser feito a partir da tabela de produção do SIA - SUS, com valores difarenciados e de um valor fixo repassado pelo MS para a implantação das equipes ${ }^{28,29}$.

A implantação deste modelo em Porto Alegre iniciou-se em dezembro de 1995, com a assinatura de um convênio entre o MS e a Secretaria de Saúde. Este convênio repassou um milhão de reais para a implantação de 30 equipes do programa no município. $\mathrm{O}$ convênio previa a implantação de 100 equipes que deveriam ser localizadas onde não existiam serviços de saúde para atender principalmente as populações mais carentes. Os critérios de escolha da localização das equipes que foram efetivamente implantadas foram: (a) a dificuldade de acesso a outros equipamentos de saúde; (b) o perfil sociossanitário das comunidades. Assim, as equipes estão em comunidades pobres que apresentam o perfil de morbidade complexo, com prevalência de doenças crônicas e agudas. A população atendida é de 100.818 pessoas, havendo em média 3.600 pessoas por equipe. O PSF cobre em torno de $8 \%$ da população do município

Neste estudo, o nosso objetivo foi estudar a estrutura de custos do PSF de Porto Alegre, identificando as tendências na aplicação dos recursos e a evolução dos custos dos serviços nos anos de 1998 e 2002.

\section{Materiais e Métodos}

Para avaliar os custos da Atenção Básica provida pelo Programa de Saúde da Família propomos a metodologia de análise de custos por absorção $\mathrm{O}^{30,31,32}$ e procuramos utilizar os dados disponíveis na Secretaria da Saúde, sem que se impusesse o custo da produção de novas informações, mas apenas a organização dos dados existentes. No entanto, algumas dificuldades metodológicas se apresentaram, especialmente em relação às diversas mudanças introduzidas no período de quatro anos, tanto pelo Ministério da Saúde (MS), quanto pela Secretaria Municipal de Saúde (SMS). Essas alterações exigiram a reformulação e readequação das estratégias de coleta e organização dos dados, criando algumas dificuldades na comparação entre os períodos.

O primeiro problema foi em relação aos tipos de procedimentos considerados - no ano de 1998, a tabela de pagamentos do MS era muito mais simplificada e tinha uma especificação muito menor em relação ao tipo de atendimento realizado do que em relação a tabela de procedimentos de 2002. A solução encontrada foi fazer uma comparação a partir de grandes grupos; criamos quatro deles, assim nomeados: (a) Procedimentos (todos os atendimentos exceto consultas médicas); (b) consultas médicas; (c) não-cadastrados; (d) total de atendimentos (somatório dos três grupos). Não foram consideradas as consultas odontológicas, pois não existiam nas unidades avaliadas em 1998.

O segundo problema foi em relação às formas de transferências de recursos financeiros do MS para as SMS - em 1998 o cálculo era feito a partir da produção de cada unidade de saúde e multiplicado pelo valor dos procedimentos na tabela do SUS; em 2002 o município recebia por tetos financeiros e a solução encontrada foi, primeiro, estimar, a partir do valor recebido pelo Piso da Atenção Básica (PAB Fixo), o valor correspondente à população coberta pelo PSF e, em seguida, somar os valores recebidos por: Incentivo Adicional do Programa de Agentes Comunitários da Saúde (PACS) e PSF.

Outra questão foi em relação à depreciação dos bens imóveis: não tínhamos conhecimento dos bens adquiridos no período intermediário, 1999, 2000 e 2001; portanto, não havia como calcular a depreciação deste período. A solução foi não usar os dados da depreciação. Fizemos esta escolha com segurança, pois, nas observações feitas no estudo realizado em $1998^{1}$, percebemos que essa informação tem uma influência insignificante nos custos totais.

Com relação ao estabelecimento dos custos indiretos, também houve alterações, mas para melhor: a SMS modificou completamente a estrutura de seu orçamento e, atualmente, o orçamento permite visualizar informações de cada unidade orçamentária e atividade de uma forma detalhada, como não era possível em 1998. Sendo assim, 
foi possível estabelecer com muito mais eficiência aqueles custos que estão vinculados a todos os serviços e, portanto devem ser rateados. Escolhemos novamente o critério de número de funcionários, pois acreditamos que a quantidade de pessoas envolvidas em um determinado setor expressa os custos que gera.

Um exemplo seriam os custos de pessoal e matériaprima para produzir determinado bem ou serviço. Por outro lado, os custos indiretos são aqueles que não são identificados apenas com um produto ou serviço, mas com mais de um ou com todos. Os custos indiretos devem ser rateados entre todos os produtos e serviços produzidos. Um exemplo seriam os custos de aluguel, energia e combustível. Ver nas planilhas 1, 2 e 3 como ambos são tratados neste estudo.

O pagamento do pessoal também se alterou significativamente: em 1998 os funcionários eram contratados pelas associações de moradores e podíamos ter a informação detalhada por tipo, por mês e por unidade de saúde. Atualmente temos a informação do repasse mensal para o pagamento do pessoal. Infelizmente, não podemos mais identificar, por unidade de saúde, o custo de pessoal decorrente da rotatividade, pois somos obrigados a trabalhar com médias.

Neste artigo utilizamos apenas as informações anuais, ou seja, não faremos análise do comportamento mensal das variáveis. Além disso, optamos, por questões metodológicas, trabalhar apenas com as 22 unidades do PSF que existiam nos dois anos estudados e que são compostas por apenas uma equipe de saúde cada uma. Assim o nosso centro de custo final é igual à unidade de saúde, que, por sua vez, é igual a uma equipe do PSF.
Foram excluídas da análise as unidades antigas que passaram a contar com duas ou mais equipes do PSF em 2002 e, também, as unidades criadas após 1998. Atualmente a SMS possui 57 unidades de saúde do PSF, algumas delas com mais de uma equipe por unidade. Em 1998 as unidades eram compostas por apenas uma equipe do PSF, com uma exceção que possuía uma equipe ampliada (dois médicos e três auxiliares). Estas unidades atendiam em média 3.281 pessoas por equipe. Em 2002 cada equipe do PSF adota um nome e existem, eventualmente, mais de uma equipe numa mesma unidade, criando nomes tipo Esmeralda I e II. Neste período foram criadas mais seis equipes (Castelo II, Esmeralda II, Jardim Cascata II, Morro dos Sargentos II, Ponta Grossa II e Santa Teresa II) nas unidades “antigas” e os usuários foram redistribuídos entre elas. Em função dessa escolha, de trabalharmos apenas com a equipe original e de termos excluído aquelas que foram duplicadas, tivemos um número de usuários por equipe menor - diminuiu para 3.007 pessoas em média. Os dados financeiros foram atualizados para dezembro de 2002 com base no Índice Nacional de Preços ao Consumidor (INPC), Instituto Brasileiro de Geografia e Estatística (IBGE).

\section{Resultados e Discussão}

Como resultados, vimos, em primeiro lugar, que o custo total anual per capita das 22 unidades do PSFPOA aumentou no período em torno de 33,51\% (Figura 1). Mas, ao mesmo tempo, é interessante observar que o custo médio anual dos atendimentos, aqui considerando todos os atendimentos realizados e os exames solicitados, manteve-se constante neste período, em torno de 18 reais

Planilha 1. Informações gerais sobre o Programa de Saúde da Família. Porto Alegre, 1998 e 2002.

\begin{tabular}{lcccc}
\hline & \multicolumn{2}{c}{1998} & \multicolumn{2}{c}{2002} \\
Informações gerais / Anos & No. & Percapta & No. & Percapta \\
\hline Usuários Cadastrados & 72.186 & NA & 66.163 & NA \\
Número de exames solicitados & 32.744 & 0,45 & 92.552 & 1,4 \\
A. Total de procedimentos exceto consultas médicas & 256.560 & 3,55 & 356.604 & 5,39 \\
B. Total de consultas médicas & 79.962 & 1,11 & 118.517 & 1,79 \\
Total de atendimentos (A+B) & 337.400 & 4,67 & 475.121 & 7,18 \\
\hline
\end{tabular}

Fonte: Secretaria Municipal de Saúde de Porto Alegre 
Planilha 2. Informações sobre receitas, despesas e custos do Programa de Saúde da Família. Porto Alegre, 1998 e 2002.

\begin{tabular}{|c|c|c|c|c|}
\hline \multirow[b]{2}{*}{ Informações gerais / Anos } & \multicolumn{2}{|c|}{1998} & \multicolumn{2}{|c|}{2002} \\
\hline & $\mathbf{R} \$$ & Percapta & $\mathbf{R} \$$ & Percapta \\
\hline A. Custos diretos $(\mathrm{a}+\mathrm{b}+\mathrm{c}+\mathrm{d})$ & $5.366 .751,29$ & NA & 7.982.007,96 & NA \\
\hline a. Material de consumo & $1.063 .359,91$ & NA & 1.413.099,56 & NA \\
\hline b. Outros serviços e encargos & $116.984,05$ & NA & 331.751 .92 & NA \\
\hline c. Pessoal & $3.991 .644,37$ & NA & $5.716 .550,11$ & NA \\
\hline d. Exames e diagnósticos solicitados & $194.762,96$ & NA & $520.613,00$ & NA \\
\hline B. Custos indiretos (rateios) & $938.210,81$ & NA & $742.180,73$ & NA \\
\hline Custo total $(\mathrm{A}+\mathrm{B})$ & $6.304 .962,10$ & 87,34 & 8.724.198,69 & 131,86 \\
\hline Receita do SUS (R\$) & $683.978,62$ & 9,48 & $1.691 .955,34$ & 25,57 \\
\hline Índices - INPC - IBGE & 1,469 & & 1,1474 & \\
\hline
\end{tabular}

Fonte: Secretaria Municipal de Saúde de Porto Alegre

Planilha 3. Indicadores do Programa de Saúde da Família. Porto Alegre, 1998 e 2002.

\begin{tabular}{lll}
\hline & $\mathbf{1 9 9 8}$ & $\mathbf{2 0 0 2}$ \\
Indicadores / Anos & $\mathbf{0}$ & $\mathbf{\%}$ \\
\hline Custo pago com receita federal & 10,85 & 19,39 \\
Custo direto em relação a custo total & 85,12 & 91,49 \\
Custo indireto em relação a custo total & 14,88 & 8,51 \\
\hline Índices - INPC - IBGE & 1,469 & 1,1474 \\
\hline
\end{tabular}

Fonte: Secretaria Municipal de Saúde de Porto Alegre

para os atendimentos e 6 reais para os exames, em valores atualizados pelo INPC-IBGE. Podemos presumir que a quantidade de atendimentos e de exames aumentou no período, o que de fato ocorreu - em 1998, foram realizados 4,67 atendimentos per capita, e, em 2002, foram 7,18, correspondente a um aumento de 53,75\% na quantidade de atendimentos realizados. Sendo que, destes atendimentos, as consultas médicas $(83 \%)$ foram as que mais cresceram os outros procedimentos aumentaram menos (60\%). O mesmo aconteceu com os exames, que passaram de 0,45 para 1,40 exames solicitados per capita. Acreditamos que é possível explicar a maior eficiência do gasto com a produção de um número maior de procedimentos e a manutenção de seu custo médio, em função da estabilização da gestão das unidades, considerando que, em 1998, estavam completando apenas um ano de criação, e, passados quatro anos, em 2002, houve tempo suficiente para organizar a gestão das unidades.

Figura 1. Receita Federal e custo total per capita de 22 unidades de saúde do Programa de Saúde da Família. Porto Alegre, 1998 e 2002.

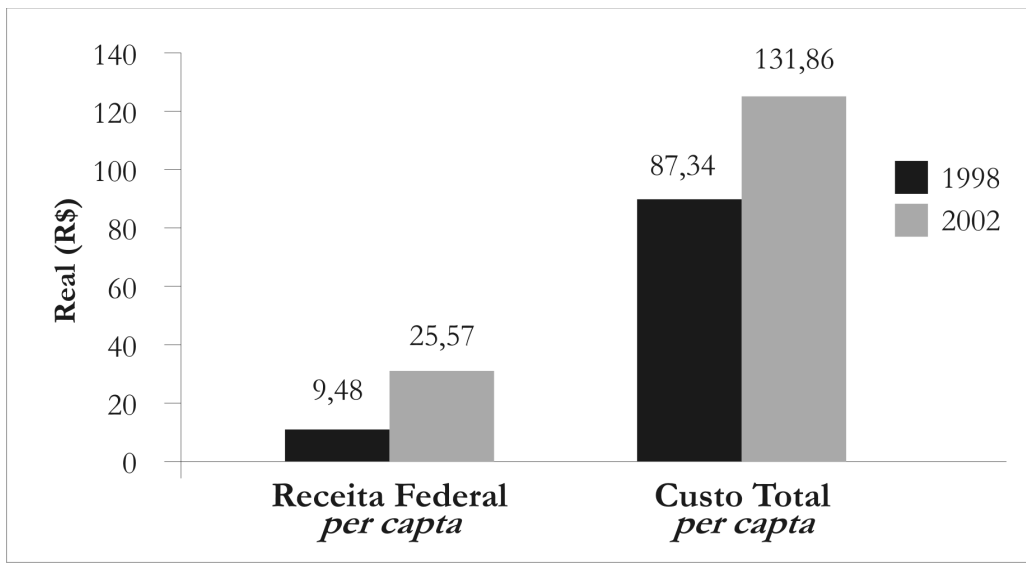


Ainda com base na Figura 1, vamos analisar as receitas: aqui consideramos os recursos de origem federal - como podemos observar, mantêm-se na mesma tendência, mas com uma ligeira melhora para os municípios. Os recursos são insuficientes para o financiamento do PSF. Em 1998 os recursos de origem federal pagavam apenas 11\% dos custos e em 2002 era possível pagar 19,39\%. Houve, portanto, um aumento da parcela financiada pelo governo federal, mesmo que ainda a responsabilidade maior com o financiamento do PSF recaísse sobre o município $(80 \%)$.

Em relação à participação dos custos diretos e indiretos nos custos totais, houve um aumento da participação dos custos diretos que passou de $85 \%$ do total, em 1998, para 91\% do total - esta alteração é uma decorrência das alterações realizadas na estrutura do orçamento da SMS que permitiram identificar com maior precisão as despesas. Os custos indiretos em 2002 representam apenas 9\% dos custos totais.

Por fim, com relação aos tipos de despesas e sua importância, a despesa com pessoal permanece a mais significativa, passando de 63,31\% em 1998 para 65,53\% em 2002. As despesas com exames e diagnósticos aumentaram de $3 \%$ para $6 \%$, enquanto a despesa com material de consumo sofreu uma redução de $16,87 \%$ para $16,20 \%$; com outros serviços e encargos, um aumento de 1,85\% para 3,80\% da despesa total.

Enfim, não pretendemos apresentar conclusões, mas retomar e aprofundar algumas idéias que consideramos relevantes para discutir a avaliação de custos da atenção básica em saúde. Nos últimos anos, o sistema de saúde brasileiro vem passando por diversas reformulações, dentre elas, a descentralização das ações de saúde para estados e municípios - sendo que os últimos assumiram as principais responsabilidades com a prestação de serviços de saúde. Este fato levou os gestores a iniciarem diversas ações de reorganização dos serviços de saúde. Um dos desafios é reestruturar a rede básica de atenção à saúde, sem reduzir a oferta de serviços, garantindo o acesso universal e integral, com a hierarquização dos níveis da assistência. O gerenciamento dos custos da assistência é uma alternativa para que se realizem estas reformas, sem a necessidade de redução da oferta de serviços, pois, por meio do gerenciamento dos custos, é possível realizar uma gestão mais eficiente. Nesta medida, o instrumental econômico é utilizado para a identificação dos problemas e para a criação de alternativas para a sua superação.

Sabemos que existem profundas diferenças entre a forma de organização e de funcionamento do setor público e do setor privado. Sabemos, também, que os sistemas de custeio foram criados para aumentar a competitividade das empresas privadas - cujo principal objetivo é o lucro -, enquanto que as instituições do setor público têm a função social como o seu principal objetivo. No entanto, não significa que não é necessário estudar o custo das atividades de atenção à saúde para melhorar a eficiência do gasto público e modificar a estrutura de custos das secretarias de saúde, se necessário.

Sugerimos que mais trabalhos desta natureza sejam realizados, por um número maior de municípios. Desta forma será possível identificar as diferenças e semelhanças entre eles, estabelecer alguns parâmetros importantes de custos e aprimorar os indicadores.

Resumindo, podemos afirmar que o conhecimento das estruturas de custos das instituições de saúde é fundamental para que o gestor possa viabilizar algumas alterações no modelo de atenção que poderão garantir maior universalidade e integralidade da atenção.

\section{Referências}

1- Castro JD. A utilização do sistema de custeio por absorção para avaliar custos da atenção básica de saúde: reformulações e aprimoramentos metodológicos. [Tese]. Campinas: Unicamp; 2000.

2- Mcguire A, Henderson JB, Mooney G. The economics of health: an introdutory text. London: Routledge and Kegan Paul; 1992.

3- Culyer AJ. The economics of social policy. London: Martin Robertson; 1973.

4- Normand C. Economics, health and the economics of health.. Br Med J 1991; 303:21-23. 
5- Abel Smith, March EP. Planning the finances of the health sector: WHO; 1983.

6- Banco mundial. Relatório sobre o desenvolvimento mundial 1993: investindo em saúde. Rio de Janeiro: Fundação Getúlio Vargas; 1993.

7- Barr N. Economics of the welfare state. London: Weindenfield \& Nicolson; 1987.

8- Culyer AJ. Health, health expenditure and equity [Discussion Paper,83]. York: University of York; 1991.

9- Hartz MA. Avaliação em saúde: dos modelos conceituais à prática na análise da implantação de programas. Rio de Janeiro: Fiocruz; 1997.

10- Hartz MA, Pouvouville G. Avaliações dos programas de saúde: a eficiência em questão. Ciênc. saúde coletiva. 1998;7(1):68-82.

11- Berman HJ, Weeks LE. Administração financeira de hospitais. São Paulo: Pioneira; 1979.

12- Matos AJ. Manual de custos hospitalares: uma metodologia simplificada. [s.l]: Federação Brasileira de Hospitais; [s.d.]

13- Brasil, Ministério da Saúde. Manual de apuração de custos hospitalares. Brasília: Centro de Documentação do Ministério da Saúde; 1988.

14- Conselho Regional de Contabilidade do Estado de São Paulo. Custo como ferramenta gerencial. São Paulo: Atlas; 1995.

15- Federação Brasileira de Hospitais. Manual de custos hospitalares: uma metodologia simplificada. [s.l.] FBH [s.d.]. 16- Lima MG. Avaliação de custos do tratamento hospitalar de esquizofrenia: um estudo retrospectivo. [Dissertação]. Campinas: Unicamp; 1997.

17- Gauthier PH, Grenon A, Pinson G, Rameau E. O controle de gestão no hospital. São Paulo: Centro São Camilo de Desenvolvimento em Administração da Saúde - CEDAS; 1979.

18- Médici A, Marques R. Sistemas de custos como instrumento de eficiência e qualidade dos serviços de saúde. São Paulo: FUNDAP/IESP; 1995.

19- Beulke R, Bertó DJ. Gestão de custos e resultados na saúde: hospitais, clínicas, laboratórios e congêneres. São
Paulo: Saraiva; 1997.

20- Piola S, Vianna S. Economia da saúde: conceitos e contribuições para a gestão em saúde. Brasília: IPEA; 1995. 21- Silva Junior AG. Modelos tecnoassistenciais em saúde: o debate no campo da saúde coletiva. São Paulo: Hucitec; 1998.

22- Campos GS. A saúde pública e a defesa da vida. 2.ed. São Paulo: Hucitec; 1994.

23- Castro MHG. Descentralização e política de saúde: tendências recentes. Planej polít Públicas 1991;(5):105-121. 24- Cecílio L. A construção de uma cultura institucional de planejamento: a contribuição do município. Saúde Debate. Mar. 1991; (31):67-71.

25- Rivera JU, Matus C, Testa,M. Planejamento e programação em saúde: um enfoque estratégico. São Paulo: Cortez; 1989.

26- Schraiber IB. Programação em saúde hoje. 2.ed. São Paulo: Hucitec; 1993.

27- Schraiber LB. Políticas públicas e planejamento nas práticas de saúde. Saúde Debate. Jun. 1995;47:28-35.

28- Brasil. Ministério da Saúde. Construindo um novo modelo. Saúde da família. 1996; 1(1).

29- Brasil. Ministério da Saúde. Saúde da família: uma estratégia de organização dos serviços de saúde. Brasília (DF): Documento preliminar; 1996.

30- Instituto de Pesquisa Econômica Aplicada. Manual de elaboração e análise de projetos sociais. Brasília (DF): [s.n]; 1996.

31- Nakagawa M. Introdução a controladoria: conceitos, sistemas, implementação. São Paulo: Atlas; 1995.

32- Oliveira ET, Pacheco MJ. Custos e finanças no hospital: uma abordagem gerencial. São Paulo: CEDAS; 1986.

\section{Endereço para correspondência:}

Janice Dornelles de Castro

Furriel Luis Antonio Vargas 106/404

Porto Alegre - RS 90470-130

\section{Endereço eletrônico}

janicedc@via-rs.net 